\title{
Best-Practice Coaches: An Untapped Resource in Sport-Science Research
}

An individual's performance development is regulated by a complex interaction of multiple factors. Our understanding of this interaction is far from complete, something that is likely part of the reason why intuition, experience, and tradition underpin the training and coaching of elite athletes to a greater extent than research-based evidence. Numerous scientific investigations over the years have been devoted to understanding the training and physiology of world-leading competitors. However, behind every successful athlete stands a coach. History has shown that the very best coaches often are years ahead of sport science in employing the critical features of training, including prescription, execution, individualization, periodization, organization, application of training methods, athlete profiling, technique supervision, feedback, tapering, and tactics. ${ }^{1-4}$

The gap between best practice and science grabbed my awareness in the early 1990s when I was a bachelor's student and mediocre athletic sprinter. At that time, I had the privilege to practice in one of the most successful sprint groups in Europe, led by the Norwegian national-team sprint coach Leif Olav Alnes. My sprinting career is not worth mentioning, but the practical knowledge gained by observing, assisting, and participating in Norwegian athletics through the development of several elite sprinters has been invaluable for my later academic career. Today, 30 years later, Leif Olav is still going strong, coaching, among others, the 400-m hurdler Karsten Warholm. Under the wing of Leif Olav since 2015, Warholm has so far improved his personal best every year, from 51.09 seconds to a new world record of 46.70 seconds (as of July 1 , 2021). While most world-leading sprinters and hurdlers improve their personal bests by $\sim 1 \%$ in the 5 years preceding peak performance (ie, $\sim 0.2 \%$ each year), ${ }^{5}$ Warholm has so far improved his performance by more than $8 \%$ since the age of 19 . Obtaining the knowledge behind the training process leading to such a giant leap in performance would be priceless for coaches working with sprinting athletes.

During the 1950s, New Zealander Arthur Lydiard (coach of Peter Snell and Murray Halberg) broke with contemporary middleand long-distance practice by prescribing a large volume of lowintensity running to his athletes during the preparation period, while the amount of high-intensity training was introduced gradually as the competition season approached. Lydiard's training organization model is still valid, although refinements have been made over the years. Today, the training of Henrik, Filip, and Jakob Ingebrigtsen (three Norwegian brothers, all European 1500-m champions) gains attention in the middle-distance community, in which a larger proportion of their interval training seems to be performed at (not above) anaerobic threshold than is usual in contemporary practice. Other athletes have now started to adapt this training organization, likely creating a shift in current bestpractice training recommendations. Another example of coach leadership, this time in a team sport, is the results and achievements of Manchester United Football Club under the leadership of Alex Ferguson, winning 38 trophies over a 26-year period (including 13
Premier League titles, 5 FA Cups, and 2 UEFA Champions League titles).

To capture the knowledge derived from best coaching and scientific practice, IJSPP aims to publish retrospective analysis of the training prescribed or performed by leading practitioners, prospective studies monitoring the longitudinal development of high-level athletes, and experiments or interventions (based on best-practice training) exploring the underlying mechanisms. In cases where the mysteries and secrets of the training process are challenging to quantify, an integrative and/or multidisciplinary approach may be required to capture a larger proportion of the knowledge underpinning the performance development. In this way, science will improve practice, and vice versa.

It is somewhat surprising that learning from the very best coaches has received minimal attention in sport-science literature. A review of all original investigations and brief reports published in IJSPP from 2015 to 2021 (more than 1100 studies) reveals that coaches have been recruited as participants in less than $0.5 \%$ of the cases. Plausible reasons for this unexploited potential source might be related to lack of time and priority among coaches, uncertainty regarding what criteria make a good coach, and lack of verified methodology on how to systematize knowledge from coaches in our field. It is often hard to differentiate a great or good coach from an average one, as many coaches have achieved success despite limited experience and education. Many medium talents have been destroyed by coaches who tried to apply a program that worked well for a champion athlete. The successful sprint coach Leif Olav Alnes use to say that "it takes a hell of a bad coach to ruin a good athlete." Unarguably, success with multiple athletes over time is a strong criterion for being considered a good coach. It is always challenging to distinguish general beliefs and scientific facts, and history has shown that both scientific and experiencebased "truths" have often only had temporary validity. The training science community should therefore strive to describe and verify what the best coaches claim to have known for a long time, and at the same time embrace coaches who have the guts to question research work in their field. This approach provides an effective means of establishing mechanistic connections between training, competitive performance, and underlying physiological determinants.

Thomas Haugen, IJSPP Associate Editor, Kristiania University College, Norway

\section{References}

1. Storey A, Smith HK. Unique aspects of competitive weightlifting: performance, training and physiology. Sports Med. 2012;42(9): 769-90.

2. Bordonau JLD, Mendez-Villanueva J. Tactical Periodization. A Proven Successful Training Model. SoccerTutor.com Ltd; 2018. 
3. Haugen T, Seiler S, Sandbakk $\varnothing$, Tønnessen E. The training and development of elite sprint performance: an integration of scientific and best practice literature. Sports Med Open. 2019;5:44.

4. Haugen T, Sandbakk Ø, Enoksen E, Seiler S, Tønnessen E. Crossing the golden training divide: the science and practice of training worldclass 800- and 1500-m runners [Published online ahead of print May
21, 2021]. Sports Med. PubMed ID: 34021488 doi:10.1007/s40279021-01481-2

5. Haugen TA, Solberg PA, Foster C, Morán-Navarro R, Breitschädel F, Hopkins WG. Peak age and performance progression in world-class track-and-field athletes. Int J Sports Physiol Perform. 2018;13(9): 1122-1129. doi:10.1123/ijspp.2017-0682 